\title{
NEUROSCIENCE
}

\section{Optimizing AAV delivery to the brain}

Goertsen, D., Flytzanis, N.C., Goeden, N. et al. Nat. Neurosci. 25, 106-115 (2022)

Gene therapy is rapidly emerging as a powerful therapeutic strategy for neurodegenerative disorders, such as Alzheimer's disease, Parkinson's disease and Huntington's disease. Adeno-associated virus (AAV) - a small virus that can be engineered to deliver DNA to target cells - is one of the most promising vehicle for gene therapy targeting the central nervous system (CNS). Even though intravenous (IV) delivery of AAVs is noninvasive and technically feasible, most AAV-based gene therapies rely on serotypes that have a broad tropism for different cell types, limiting the efficacy of AAV systemic injection for CNS-directed gene therapy.

In a new study, a research team led by first authors David Goertsen, Nicholas Flytzanis and Nick Goeden, from the Gradinaru lab at the California Institute of Biology, engineered AAV capsid variants that selectively target neurons after IV delivery in mice and marmosets. "These results constitute an important step forward toward achieving the goal of engineered AAV vectors that can be used to broadly deliver gene therapies to the CNS in humans," write the investigators in their report.

To identify novel variants with tissueand cell-specific tropism, the investigators applied M-CREATE, a selection method that combines the generation of a library of AAV capsid sequences that contain a polyadenylation sequence flanked by Cre-Lox sites, production of viruses from the DNA library, injection of the AAV library in different transgenic mice expressing Cre recombinase in specific tissues, and next-generation sequencing (NGS) to screen viral variants across the mice. With this approach, PCR amplification occurs after Cre-Lox recombination, only for the variants that have successfully transduced $\mathrm{Cre}^{+}$cells. This method enables the recovery of unique sequences with a desired transduction profile.
After two rounds of selection, the researchers identified 39,034 sequences that resulted in CNS enrichment and decreased targeting of the liver, and they selected 22 AAV variants for further testing in vivo. The different viral variants, modified to carry a fluorescent reporter gene, were injected intravenously in wild-type mice, which were imaged after few weeks. Fluorescence quantification identified six variants with high fluorescence in the brain and limited targeting of the liver, including one variant exhibiting high specificity to neurons. The tropism of the variants was confirmed in marmosets, in which AAVs enabled robust gene delivery to the brain after IV administration.

\section{Alexandra Le Bras}

Published online: 2 February 2022 https://doi.org/10.1038/s41684-022-00924-1



\section{How to work smarter in the new normal}

Sign up for a free short email series with top tips, resources and advice for those starting or returning to research, brought to you by the Nature Briefing team.

\section{go.nature.com/backtothelab}

